\title{
Adaptive platform for fluorescence microscopy-based high-content screening
}

\author{
Matthias Geisbauer ${ }^{a}$ and Thorsten Röder ${ }^{b}$ and Yang Chen ${ }^{b}$ and Alois Knoll ${ }^{b}$ and Rainer Uhl ${ }^{a}$ \\ ${ }^{a}$ BioImaging Zentrum of Ludwig-Maximilians-Universität, \\ Großhaderner Str. 2-4, 82152 Martinsried, Germany; \\ ${ }^{b}$ Technische Universität München, \\ Boltzmannstr. 3, 85748 Garching bei München, Germany
}

\begin{abstract}
Fluorescence microscopy has become a widely used tool for the study of medically relevant intra- and intercellular processes. Extracting meaningful information out of a bulk of acquired images is usually performed during a separate post-processing task. Thus capturing raw data results in an unnecessary huge number of images, whereas usually only a few images really show the particular information that is searched for. Here we propose a novel automated high-content microscope system, which enables experiments to be carried out with only a minimum of human interaction. It facilitates a huge speed-increase for cell biology research and its applications compared to the widely performed workflows. Our fluorescence microscopy system can automatically execute application-dependent data processing algorithms during the actual experiment. They are used for image contrast enhancement, cell segmentation and/or cell property evaluation. On-the-fly retrieved information is used to reduce data and concomitantly control the experiment process in real-time. Resulting in a closed loop of perception and action the system can greatly decrease the amount of stored data on one hand and increases the relative valuable data content on the other hand. We demonstrate our approach by addressing the problem of automatically finding cells with a particular combination of labeled receptors and then selectively stimulate them with antagonists or agonists. The results are then compared against the results of traditional, static systems.
\end{abstract}

Keywords: fluorescence microscopy, high-content, sample screening, live cell analysis, DSP, FPGA, computer vision, closed-loop system

\section{MOTIVATION}

High content-screening is the attempt to obtain large, statistically relevant data sets from live cells in order to answer sophisticated questions previously asked in research institutions only. Besides transillumination many other techniques, such as fluorescence resonance energy transfer (FRET), fluorescence recovery after photobleaching (FRAP), total internal reflection fluorescence (TIRF) or laser scanning have been developed throughout the years. In recent time cell biology and the corresponding fluorescence microscopy techniques more and more find their way into drug discovery. ${ }^{1,2}$ Besides high-throughput screening where more than 100,000 samples are tested per day, using rather simple techniques, complex single cell experiments are attempted on a larger scale today. ${ }^{3}$ During the last years more and more live cell assays have been developed for high-content drug screening and it has been shown that fluorescence microscopy is a powerful tool for acquiring multiparametric data. ${ }^{4-6}$ Recent trends in high-content screening shift experiments to higher spatial and temporal resolutions. In order to achieve high-content, automated microscopes have to integrate many more imaging modes than are available in existing equipment. ${ }^{7}$ The main drawback of fluorescence microscopy in high-content screening is the huge amount of data that gets collected within a short time. Due to the desired high resolution and the unevenly distributed samples, the amount of useless data without any useful information is likewise increased and therefore renders a big penalty for fluorescence microscopy in high-content screening.

Nowadays, the amount that needs to be stored is likely to reach the terabyte dimension during a single day of the experiment. By doing this the intermediate data, collected during the biological experiment, needs to be stored

E-mail: geisbauer@biz.uni-muenchen.de, roeder@in.tum.de, yang.chen@in.tum.de, knoll@in.tum.de, uhl@biz.unimuenchen.de 
on a temporary or permanent storage system. For separated post-processing this data is often stored as raw data, causing the need for high-capacity and fast storage systems. This large amount as well as the increasing computational effort required to process and analyze this data constitute a performance bottleneck in today's microscopy applications. ${ }^{8}$ Due to the complexity of high-content microscopy applications, the majority of single cell experiments is still done "by hand", which results in very laborious and time-consuming workflows, limiting the experiment throughput. On the other hand, the longer duration of manual experiments is contrary to the sensitivity of living cells with respect to environmental conditions. That contradiction can only be handled if the image analysis is done in a separate post-processing phase thereby limiting the possibilities for experiment supervision or efficiency control. In this paper we propose a novel adaptive microscopy architecture for enabling the creation of intelligent high-content systems for live cell analysis, with this minimizing the needed time but increasing the content, by enhancing the image quality with image contrast enhancement (bleaching-balancing), cell segmentation (regions-of-interest) and/or cell property evaluation (pattern-recognition).

The paper is organized as follows: section 2 describes the underlying system architecture and the basic visual processing steps, section 3 gives information about applications and experimental results and section 4 concludes the proposed approach.

\section{ADAPTIVE CONTROL PLATFORM}

The adaptive system comprises a MORE microscope (TILL Photonics GmbH*, Martinsried, Germany), which is capable of performing fully automated fluorescence microscopy applications. Novel LED and laser illumination techniques combined with an extremely stable motorized X-Y stage, ultra fast dichroic and objective changers, a high precision voice-coil-based focus drive with integrated focus clamp and a pco.1600 charge coupled device (CCD) camera $\left(\mathrm{PCO}^{\dagger}\right.$, Kelheim, Germany) with a spatial sensor resolution of $1600 \times 1200$ pixels each providing a dynamic range of 14 bits at an upper frame rate of 30 fps turn the microscope system into an ideally suited system for automated high-content microscopy including many major microscopy techniques such as transillumination, FRET, FRAP, TIRF and laser scanning. The integrated, combined DSP-FPGA based control architecture, which is an enhancement of the Integration Control Unit (ICU), ${ }^{9}$ allows hardware real-time microscope control and synchronization. All integrated components such as X-Y stage, focus drive and scanners are handled on

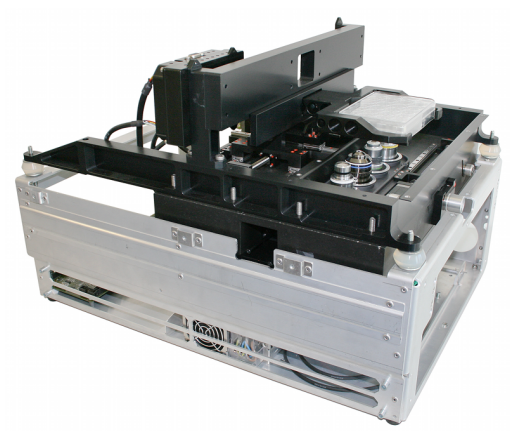

(a) MORE microscope (open frame)

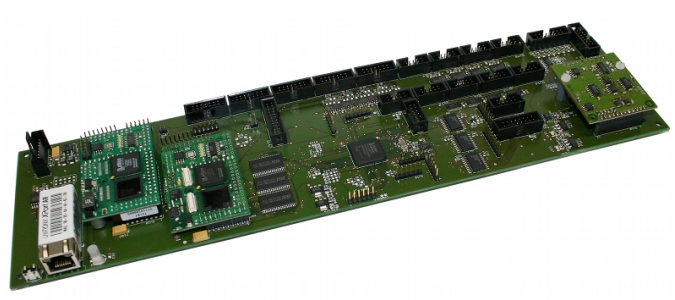

(b) Integrated Control Platform (ICP) board

Figure 1. The utilized microscope and the embedded ICP control board.

a time base of $10 \mu \mathrm{s}$. Controlling functions at which a more precise timing is important to avoid divergency in experiment conditions, like exposure time triggers or image acquisition trigger synchronization, are done on a $1 \mu \mathrm{s}$ base. Unlike the former modular microscope control system, the new integrated control platform (ICP) services all internal microscope interfaces directly with full access to all device functions, including the control of the X-Y stage, the focus drive and the CCD camera, which results in a completely independently working screening system. With a comprehensive range of interface commands for digital, analog and serial interfaces in combination with a wide variety complex system commands the ICP provides full flexibility for user-defined

\footnotetext{
*http://www.till-photonics.com

${ }^{\dagger}$ http://www .pco.de
} 
protocols. With features like loops, if-else conditions, system internal variables and a mathematics module, which are usually only known in higher programming languages, even complex protocols can be set up and then run in real-time giving the system the ability to acquire sets of statistically relevant data. Besides a hierarchical execution of real-time protocols the ICP, with its complete knowledge about the system status and a sophisticated event structure, provides the ability to adaptively modify and/or optimize the predefined experiment protocols in real-time. In order to parallelize multiple microscope platforms in screening applications a standard Ethernet interface was chosen for direct user control and/or protocol setup.

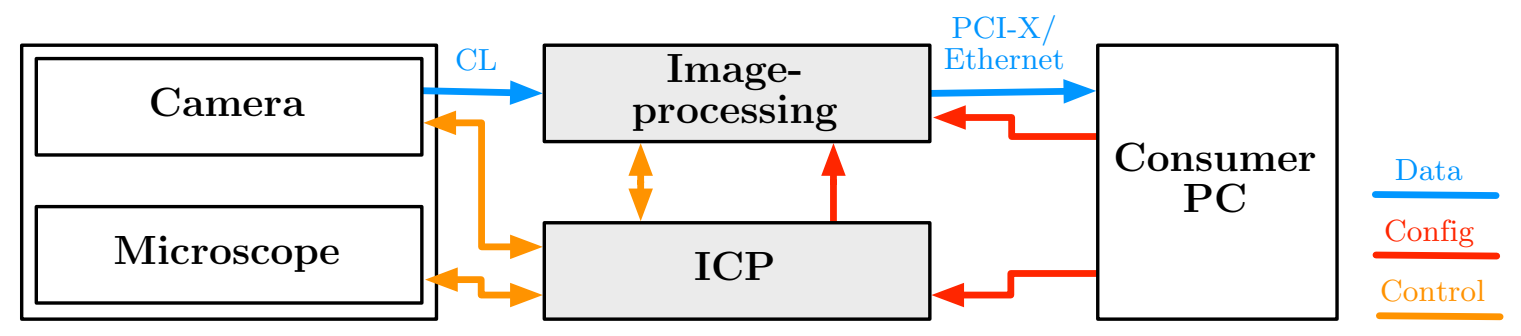

Figure 2. Hardware architecture consisting of a microscope, an integrated control platform (ICP), embedded image preprocessing unit and a consumer PC for assay configuration, monitoring, online reconfiguration and statistical evaluation. The main bandwidth is required for the camera connection, which uses a CameraLink interface (CL), and the connection to the PC, which is either a PCI-X or a gigabit Ethernet interface. The current setup communicates over the PCI-X bus. For scalability, especially when using multiple microscopes in parallel, the Ethernet standard was chosen.

Besides the control platform a programmable image data pre-processing frame grabber (Gidel ${ }^{\ddagger}$, Or Akiva, Israel), connected to the CCD via a CameraLink interface, provides the possibility of acquisition and online data interpretation. The basic hardware configuration is shown in figure 2. Coupling the data pre-processing module with the real-time environment of the ICP provides the possibility of experiment adaptation and/or optimization, not just based on system parameters only, but also based on image content analysis. The image data is interpreted during protocol execution and the gathered information is then immediately used to optimize the protocol in real-time. In order to cope with the high dimensional dynamics in a high-throughput screening system we follow a suggestion of using dedicated visual processing modules ${ }^{10}$ that are linked in a fixed processing sequence. Table 1 gives an overview of the processing modules.

Table 1. Overview of the visual processing modules and a brief description of their main tasks within the processing pipeline.

\begin{tabular}{llllr}
\hline Processing step & Module & Description & Output & Feedback \\
\hline \hline 1. & pre-processing & $\begin{array}{l}\text { processing of the } \\
\text { raw input image }\end{array}$ & normalized image & illuminance \\
\hline 2. & segmentation & cell detection & regions of special interest & region coordinates \\
\hline 3. & classification & cell recognition & cell classification & cell type \\
\hline 4. & data reduction & data reduction & lossy or lossless & compressed data \\
\hline 5. & tracking & sequential estimation & object associations & expected coordinates \\
\hline 6. & data mining & property estimation & statistical features & - \\
& & and aggregation & &
\end{tabular}

\footnotetext{
${ }^{\ddagger}$ http: //www.gidel.com
} 
The concrete implementation for each module varies and is dependent on the specific experiment requirements. Although the concrete implementation might change for a class of experiments, the overall processing order of the modules remains the same. Some assays do not require execution of all modules due to their configuration. Thus the visual processing pipeline can on the one hand be stopped at any stage and on the other hand can have omitted modules. Figure 3 depicts the implemented closed-loop control and shows the several steps that are considered in our visual processing system. It is important to mention that during a single iteration (frame) every module can access the intermediate extracted or computed results of any previously executed processing modules.

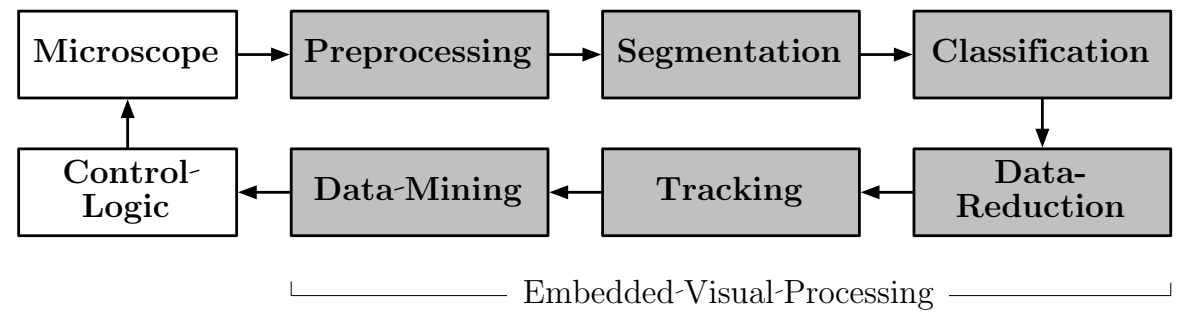

Figure 3. Visual processing components and modules: microscope (imaging device, secondary sensors and actuators), experiment logic control and visual processing and interpretation submodules.

Beginning with the pre-processing module, the captured raw camera frame is subject to static target-modelfree pre-processing. Processing based on global pixel operations could include basic flat field correction, which is especially important in light microscopy applications, due to the inhomogeneity in probe illumination and background correction or subtraction respectively.

After the pixel-wise pre-processing, the segmentation module is used to distinguish between foreground and background information by static or adaptive thresholding algorithms or optimization-based segmentation methods. Thus regions of interest (ROI), where by definition objects and not only background is localized, are defined.

In the next step the classification module takes the already found regions of interest from the segmentation module and decides if the localized objects are really objects of interest or some kind of dirt or noise. Depending on the complexity of the experiment either easy distinctions, such as thresholding or more complex algorithms such as $\mathrm{Hu}^{11}$ or Zernike ${ }^{12}$ invariant moments, template matching ${ }^{13}$ or machine learning techniques, are applied.

As the huge amount of acquired data is one of the limiting bottlenecks in high-content screening systems, a data reduction module is the next step in the image processing sequence. Data spillover minimization is done by employing selective transmission functions, which are only transmitting the selected regions of interest and not the full image or, if the whole image is required, by lossless compression.

An additional tracking module can be used to concentrate the experiment on a single cell or coherently moving cells only. This also enables cell motility estimation and can be used as a key cue to predict the near future-cell position, e.g. at the next captured frame. This requires data association for robust tracking of multi-cell targets, which is an inherent part of a multi-target tracking system.

The data-mining module is used for statistical property estimation, clustering and aggregation functions and represents the last module within the processing pipeline. The generated output is provided to the control logic unit and closes the proposed perception action loop within our system.

The implementation of the described processing modules may change according to the actual assay. Benefiting from the modular design of the visual processing system and its full flexibility of interconnection between the individual modules, the implementation of the processing pipeline can be configurated according to the specific assay in order to meet the diversifed requirements of screening experiments. In particular modules can be rearranged, cross-connected or parallelized depending on the specific requirements. Depending on the particular image processing pipeline configuration the output can be a full image, a region of interest, some discrete values which characterize the results, such as intensity values or object counts, or any possible combination of the 
above-mentioned. The used embedded visual processing system employs a FPGA-DSP hybrid architecture, which not only maximizes the performance, by ultilizing parallel computation, but also provides enough flexility to accommodate multiple different implementations, of the processing pipeline, on the same hardware.

It is important to distinguish between the output of the image processing pipeline which is transmitted and stored on the user PC and the output that is used to adapt the imaging protocol. Depending on the experiment the output of different pipeline modules can be used in order to close the feedback loop. Typical examples of feedback data are also shown in table 1.

The main advantage of the described system over conventional post-processing systems is the enormous benefit in processing time. Most processing steps are done on FPGA logic using the CameraLink pixel clock. That means that delays usually known from transferring bytes back and forth from memory to processing units are limited to just a few clock cycles. Table 2 gives an overview of the timing properties of the embedded image processing system.

\section{APPLICATION}

High-content screening applications typically demand high-throughput performance as well as at the same time semantic information evaluation. This requires efficient and fast data handling. Since storing data is also one of the major performance bottlenecks in todays systems, our proposed system tries to avoid the storage by directly extracting the desired properties online, while performing the experiment. This leads to a reduced amount of stored, and thus transmitted, data and also can provide an additional vision-based and real-time feedback channel for experiment control. Here we use our proposed system architecture for handling the inherent bleaching effect of light-exposed fluorophores and for the recording of fluorescence intensity kinetics. Hence this section will explain the implementations by referring to the previously introduced visual processing pipeline.

The bleaching effect is caused by physical constraints. To some extent, countermeasures can be applied to reduce the influences of perceived image intensity degradation. In principle two countermeasures are practically used today: the extension of the exposure time while keeping the illumination light source at constant intensity or the increase of the illumination light source intensity while keeping the exposure time constant. In order to maximize the fluorescence intensity over time, we concentrate on the first approach and increase the exposure time with diminishing fluorescence intensity.

After capture, the camera image transferred to the FPGA pre-processing module. This module operates on a raw pixel level and implements in this application a background correction by computing the one dimensional histogram of the gray level image intensities using a bin size of 20. A widely used approach in the fluorescence microscopy domain is to use this histogram to apply a background correction. By assuming that the maximum number of pixels contributing to an individual bin corresponds to the background contribution, we can use this histogram bin value $\alpha$ for background correction by subtracting this value uniformly from the image intensity. An alternative approach can be followed by using a flat-field correction ${ }^{14,15}$ approach in combination with an a-priori estimated dark frame for noise removal. By using the histogram based approach with an empirically estimated bin size, the segmentation of the image can be done analogously by thresholding the image histogram and projecting it back into the image. So either $\mathcal{I}_{n}(x, y)=\mathcal{I}(x, y)-\alpha$ or a more sophisticated flat-field correction could be applied here:

$$
\mathcal{I}_{n}(x, y)=K \frac{\mathcal{I}(x, y)-\mathcal{I}_{d}(x, y)}{\mathcal{I}_{f}(x, y)-\mathcal{I}_{d}(x, y)}
$$

where $K$ is a scaling constant, $\mathcal{I}_{n}$ is the normalized shading corrected output image, $\mathcal{I}$ is the raw input image captured by a CCD camera, $\mathcal{I}_{d}$ is the average dark frame of the camera using closed shutter and $\mathcal{I}_{f}$ is the average flat-field image in order to achieve spatial-invariant lighting conditions.

The segmentation module of our evaluation setup performs a simple intensity based thresholding decision algorithm. For this intensity cut-off we are assuming flat objects that have a suitable continuous intensity profile and neglecting cases for which the aperture problem arises:

$$
\mathcal{I}_{\tau}(x, y)= \begin{cases}1 & \text { if } \mathcal{I}_{n}(x, y) \geq \tau \\ 0 & \text { else }\end{cases}
$$


where $\mathcal{I}_{n}$ represents a normalized greyscale input image, $\mathcal{I}_{\tau}$ the respective thresholded binary image and $\tau$ the scalar threshold value.

The classification module takes the output information of the segmentation module, which currently consists of a binary image. We restrict ourselves to a simple classification scheme and interpret the two modes of the binary image, one as foreground (cell) and the other one as background (non-cell).

The data-mining module estimates the desired content properties in order to regulate the countermeasures taken according to the cell bleaching:

$$
\begin{aligned}
R_{F} & =\frac{\sum_{x, y} \mathcal{I}_{\tau}(x, y) * \mathcal{I}(x, y)}{\sum_{x, y} \mathcal{I}_{\tau}(x, y)} \\
R_{B} & =\frac{\sum_{x, y}\left(1-\mathcal{I}_{\tau}(x, y)\right) * \mathcal{I}(x, y)}{\sum_{x, y}\left(1-\mathcal{I}_{\tau}(x, y)\right)}
\end{aligned}
$$

where $R_{F}$ corresponds to the foreground intensity signal accumulation and $R_{B}$ to the background intensity signal accumulation and accordingly they are the final result for many experiments. Besides that, deviation, especially decays in foreground intensity are then used to adapt the illumination time for the following captured image. Figures 4 and 5 compare the image content of conventionally imaged HELA cells, compared to cells imaged with an adaptive illumination based fluorescence microscope. It is clearly shown that bleaching influences are minimized and image content can be kept constant.

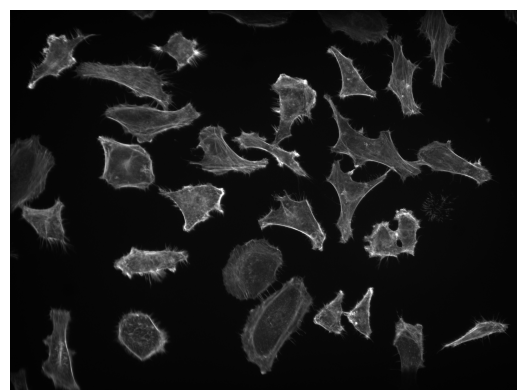

(a) Image intensity at $t_{0}=0 \mathrm{~s}$

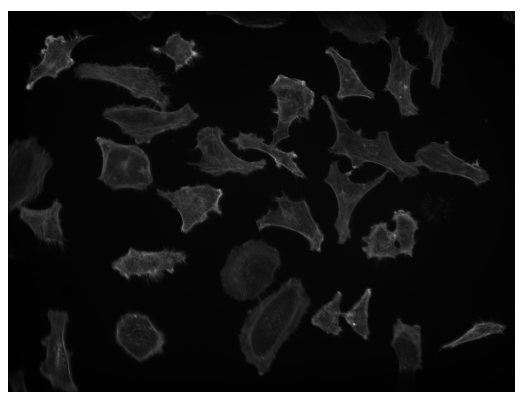

(b) Image intensity at $t_{1}=20 \mathrm{~s}$

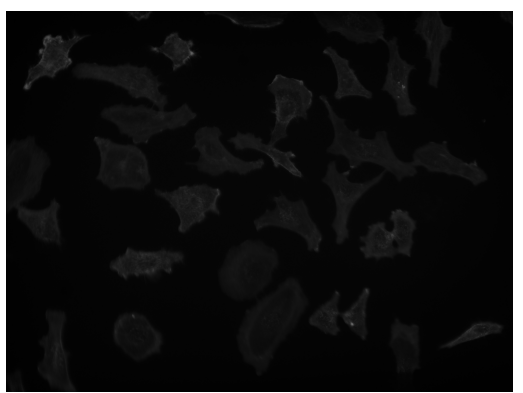

(c) Image intensity at $t_{2}=40 \mathrm{~s}$

Figure 4. Ongoing bleaching process of f-actin stained HELA cells. Pictures taken at three different timestamps with a fixed time interval of 20 s.

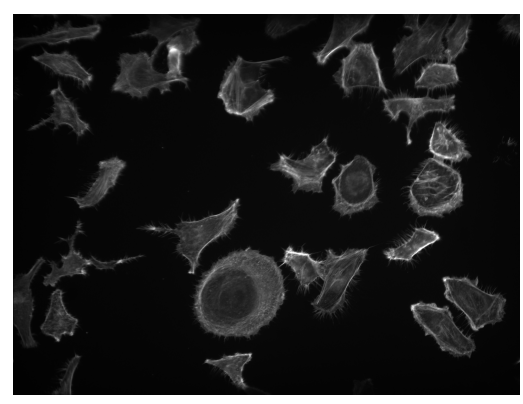

(a) Image intensity at $t_{0}=0 \mathrm{~s}$

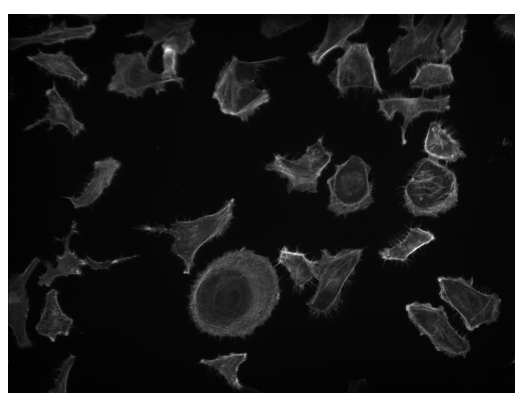

(b) Image intensity at $t_{1}=20 \mathrm{~s}$

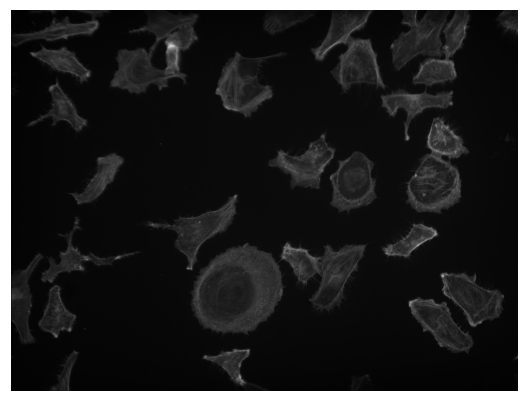

(c) Image intensity at $t_{2}=40 \mathrm{~s}$

Figure 5. Ongoing bleaching process with adapted exposure time of f-actin stained HELA cells. Pictures taken at three different timestamps with a fixed time interval of $20 \mathrm{~s}$.

The data reduction module mainly relies on the output of the classification module as shown in figure 3 . Depending on the data reduction strategy, which also relies on the microscopy experiment in general, again the goal is to reduce as much information as possible, which holds for the amount of processed and especially for the 
amount of stored data. The same described approach for estimating the intensities and regulating the applied shutter time of the camera can be used for kinetic experiments.

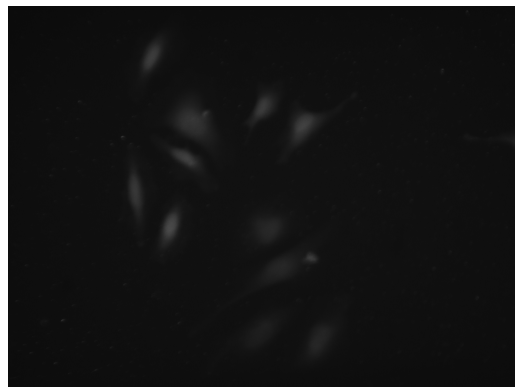

(a) Image intensity at $t_{0}=5 \mathrm{~s}$

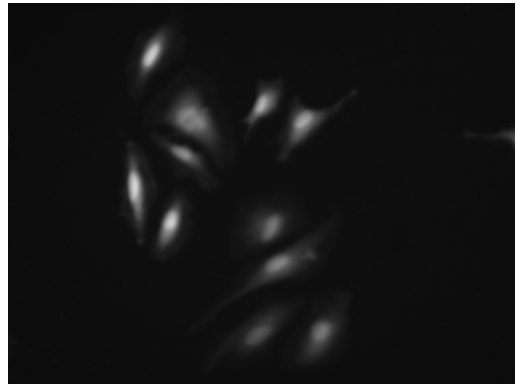

(d) Image intensity at $t_{3}=20 \mathrm{~s}$

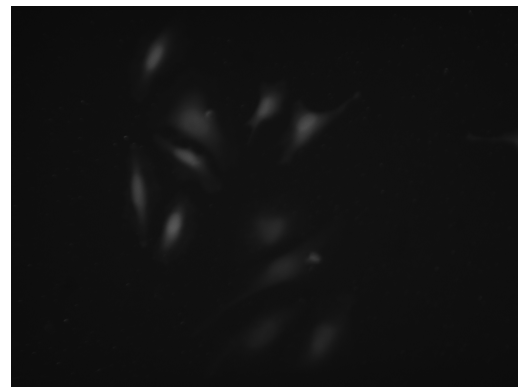

(b) Image intensity at $t_{1}=10 \mathrm{~s}$

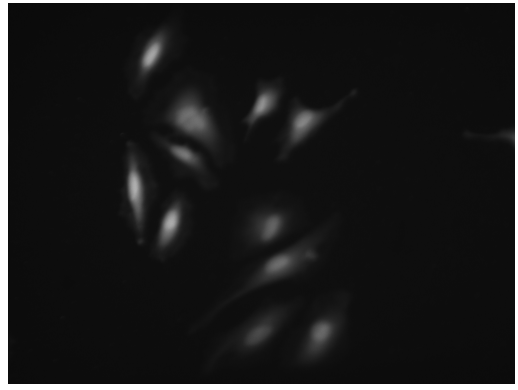

(e) Image intensity at $t_{3}=25 \mathrm{~s}$

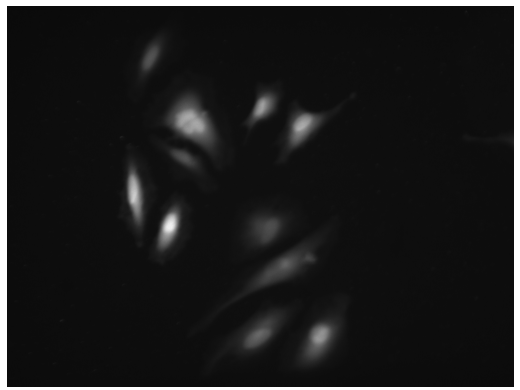

(c) Image intensity at $t_{2}=15 \mathrm{~s}$

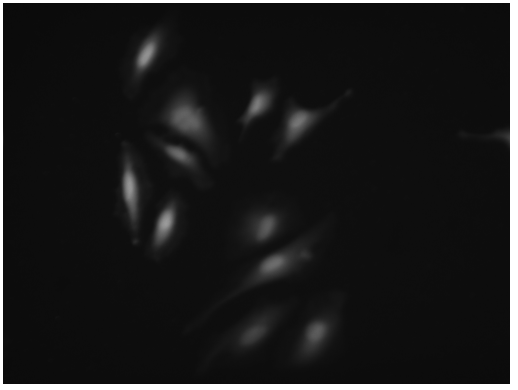

(f) Image intensity at $t_{3}=30 \mathrm{~s}$

Figure 6. Calcium kinetic of Fluo-4 stained HELA cells stimulated with ATP, 10 seconds after the beginning of the recording.

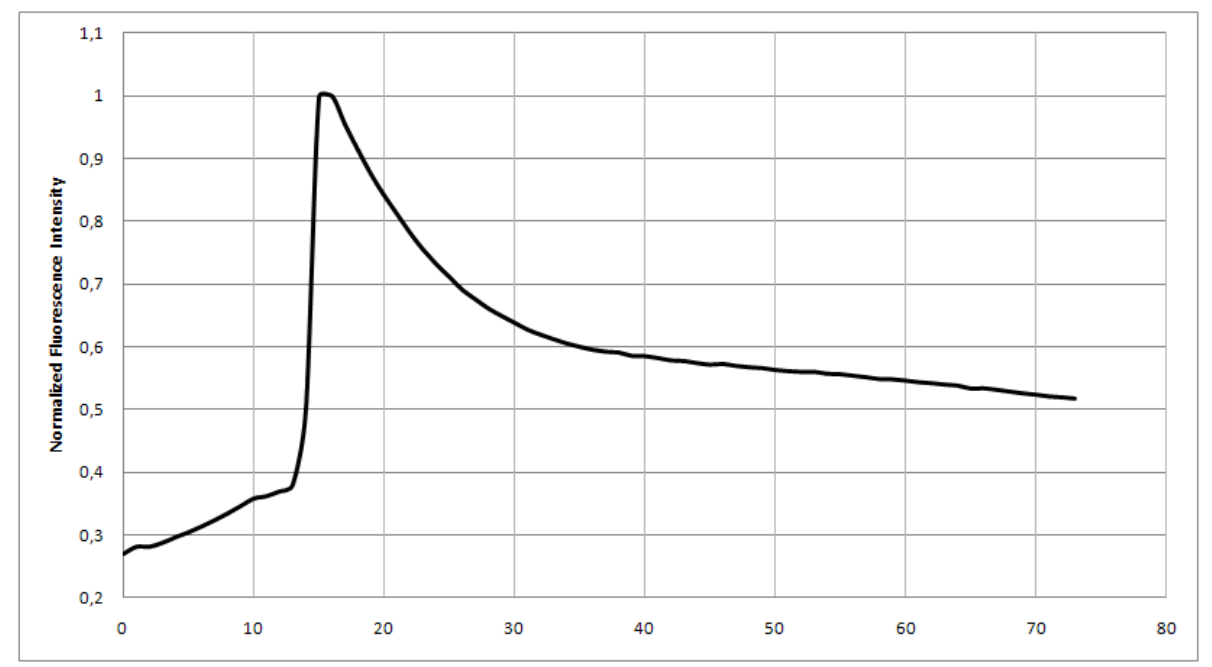

Figure 7. Calcium kinetic of Fluo-4 stained HELA cells stimulated with ATP, 10 seconds after the beginning of the recording. Normalized intensity is shown over time in seconds.

As shown in figures 6 and 7 the storage of full high-resolution images can be avoided for this kind of experiment. In fluorescence kinetic experiments for example not each single image is of particular interest, but instead only the mean fluctuation of fluorescence intensity over time needs to be estimated. Thus the proposed system allows one to adapt the computational and storage needs according to the experiment conditions by shifting the traditional 
post-processing phase into an online phase. The highly flexible and powerful platform is thus highly suited for addressing even more complex and time consuming fluorescence kinetic measurements in high-content screening. That is shown on the selective stimulation of Fluo- $4^{16}$ stained HELA cells in a high-content screening setup.

Fluo-4 is a calcium-sensitive dye, in this case used to measure fluctuations in intracellular calcium concentration. HELA cells are stained with Fluo- 4 and then measured in a transillumination setup. During imaging cells are stimulated with adenosine triphosphate (ATP), which causes an increase in intracellular calcium concentration. As calcium kinetic experiments are very time and material consuming experiments they are usually just done at a single cell base to minimize data and time overhead. Time-lapse experiments usually last up to several minutes and, depending on the desired temporal resolution acquire multiple images per second. The presented adaptive platform, with its ability to skip storing every single image, that is acquired and analyzed for recording the kinetic, further increases the temporal resolution but at the same time reduces the data volume. Figure 7 shows the full calcium kinetic with high temporal resultion and figure 6 shows a rough overview subset.

Table 2 shows that in the described high-content screening application the image processing and especially image storage is the major bottleneck. So consequently removing the need for storing the unprocessed raw data by applying automated online processing steps can significantly accelerate high-content screening using an adaptive fluorescence microcopy platform. The table also shows that the introduced processing overhead is by far smaller than the time that would be required to save each image to a state-of-the-art storage system.

Table 2. Overview of the initialization, processing and transmission times.

\begin{tabular}{lcr}
\hline Processing steps & Cycles at 80Mhz & Time \\
\hline \hline Initial camera setup & $350 \mathrm{~ms}$ \\
\hline Transmission CameraLink & $24 \mathrm{~ms}$ \\
\hline Background intensity with histogram (20bins) & 21 & $262.5 \mathrm{~ns}$ \\
Binary thresholding & 0 & $0 \mathrm{~ns}$ \\
Pixel average & 2 & $25 \mathrm{~ns}$ \\
\hline Transmission PCI overhead & $8 \mathrm{~ms}$ \\
Save image to harddrive & $220 \mathrm{~ms}$ \\
Visualization & $156 \mathrm{~ms}$ \\
\hline
\end{tabular}

\section{CONCLUSION AND FUTURE WORK}

In this paper we presented an adaptive platform for fluorescence microscopy-based high content screening that enables real-time data analysis and experiment adaptation for live cell biology. The screening system follows a combined hardware-software architecture for autonomous high-throughput assays especially targeting experiments for statistical data capturing and evaluation. The usage of an integrated FPGA/DSP combination allows the onboard early signal processing while at the same time complying with given real-time constraints. Including image pre-processing in the real-time loop not only speeds up even complex and time-consuming microscope functions, but also allows complex experiments including transillumination, FRET, FRAP, FCS, TIRF and laser scanning microscopy for high content screening. In the described applications it is shown on the one hand, that for given bleaching curves better results in terms of signal to noise can be achieved and on the other hand that the system is capable of acquiring significant kinetic data without the formerly-known image data overhead. The potential of flexibly configuring the embedded vision module makes it possible to use the system for a variety of different applications where adaptive protocols and/or online data pre-processing and reduction can be helpful or are required. This opens a completely new prospect for high-content screening and smoothes the way for high-content screening on a high-throughput scale. Furthermore we proposed a modularized embedded visual processing pipeline scheme that is at the same time flexible with respect to new processing algorithms and has high potential for parallel processing/high throughput. Including image information in the adaptive feedback loop thus is a new powerful tool for features like auto-illumination, intelligent real-time tracking or fast real-time hardware-based auto-focus functions. It follows that the system results in an optimized time-minimizing but content-maximizing microscope-based high-content embedded vision system. 


\section{ACKNOWLEDGMENTS}

The project on which this report is based was sponsored by funds from the Federal Ministry of Economics and Technology according to reference number 16IN0676. The author assumes responsibility for the content of this publication. Sponsored by BMWi on the basis of an enactment with the German Parliament.

\section{REFERENCES}

1. K. Guiliano, W. Cheung, D. Curran, B. Day, A. Kassick, J. Lazo, S. Nelson, Y. Shin, and D. Taylor, "Systems Cell Biology Knowledge Created from High Content Screening," ASSAY and Drug Development Technologies 3(5), pp. 501-514, 2005.

2. V. Starkuviene and R. Pepperkok, "The potential of high-content high-throughput microscopy in drug discovery," Br. J. Pharmacol. 152, pp. 62-71, Sep 2007.

3. S. A. Haney, P. LaPan, J. Pan, and J. Zhang, "High-content screening moves to the front of the line," Drug Discovery Today 11(19-20), pp. 889 - 894, 2006.

4. F. Gasparri, F. Sola, T. Bandiera, J. Moll, and A. Galvani, "High Content Analysis of Kinase Activity in Cells," Combinatorial Chemistry \&3 High Throughput Screening 11(7), pp. 523-536, 2008.

5. P. Denner, J. Schmalowsky, and S. Prechtl, "High-content analysis in preclinical drug discovery," Comb. Chem. High Throughput Screen. 11, pp. 216-230, Mar 2008.

6. M. Dragunow, "High-content analysis in neuroscience," Nat. Rev. Neurosci. 9, pp. 779-788, Oct 2008.

7. M. Oheim, "High-throughput microscopy must re-invent the microscope rather than speed up its functions," British Journal of Pharmacology 152, pp. 1-4, 2007.

8. Z. K. and H. S., "High content screening - the next challenge: Effective data mining and exploration," Drug Discovery World Winter 2008/09, pp. 27-34, 2008.

9. T. Geisler, J. Ressler, H. Harz, B. Wolf, and R. Uhl, "Automated Platform for High-Content and HighThroughput Analytical Screening," IEEE Trans Rob Autom 3, pp. 169-176, 2006.

10. R. Pepperkok and J. Ellenberg, "High-throughput fluorescence microscopy for systems biology," Nat. Rev. Mol. Cell Biol. 7, pp. 690-696, Sep 2006.

11. M.-K. Hu, "Visual pattern recognition by moment invariants," Information Theory, IRE Transactions on $\mathbf{8}$, pp. 179-187, February 1962.

12. A. Khotanzad and Y. Hong, "Invariant image recognition by zernike moments," Pattern Analysis and Machine Intelligence, IEEE Transactions on 12, pp. 489-497, May 1990.

13. R. Brunelli, Template Matching Techniques in Computer Vision: Theory and Practice, Wiley, har/onl ed., 52009.

14. J. A. Seibert, J. M. Boone, and K. K. Lindfors, "Flat-field correction technique for digital detectors," Medical Imaging 1998: Physics of Medical Imaging 3336(1), pp. 348-354, SPIE, 1998.

15. D. S. Lucas J. van Vliet, Frank R. Boddeke and I. T. Young, "Image Detectors for Digital Image Microscopy," in Digital Image Analysis of Microbes: Imaging, Morphometry, Fluorometry and Motility Techniques and Applications, F. S. M.H.F. Wilkinson, ed., pp. 37-64, John Wiley \& Sons, 1 ed., 51998.

16. K. Gee, K. Brown, W.-N. Chen, J. Bishop-Stewart, D. Gray, and I. Johnson, "Chemical and physiological characterisation of fluo-4 Ca2+-indicator dyes," Cell Calcium 27(2), pp. 97-106, 2000. 